\title{
Besicovitch-Eggleston Function
}

\author{
Manav Das \\ Department of Mathematics, University of Louisville, Louisville, USA \\ E-mail:manav@louisville.edu \\ Received June 2, 2011; revised July 2, 2011; accepted July 15, 2011
}

\begin{abstract}
In this work we introduce a function based on the well-known Besicovitch-Eggleston sets, and prove that the Hausdorff dimension of its graph is 2 .
\end{abstract}

Keywords: Hausdorff Dimension, Multifractal, Binomial Measure, Dyadic Intervals

\section{Introduction}

Let $x \in[0,1]$, and let $x=. x_{1} x_{2}, \cdots, x_{i}=0$ or 1 denote its binary expansion. For any $0<p<1 / 2$ we may consider the set

$$
K_{p}=\left\{x \in[0,1]: \limsup _{n \rightarrow \infty} \frac{x_{1}+x_{2}+\cdots+x_{n}}{n} \leq p\right\}
$$

Besicovitch [2] proved that

$$
\operatorname{dim}_{H} K_{p}=\frac{-p \log p-(1-p) \log (1-p)}{\log 2}
$$

where $\operatorname{dim}_{H} A$ denotes the Hausdorff dimension of the set $A$. This result was generalized to the $N$-ary case by Eggleston [8]. Billingsley proved a more general version of this result in the context of probability spaces [3]. Billingsley's result was related to densities in [5], and a similar result involving packing dimensions was proved in [6]. Sets such as $K_{p}$ are studied in the context of multifractal theory (see [1,7,9,11,14-16]) and Billingsley-type results have been proved by several authors in this context. Recently, such a result has been proved for a countable symbol space in [13].

In this paper, we are interested in a natural function that may be defined using Besicovitch's result. We call this the Besicovitch-Eggleston function: let $N[1, n, x]=\#$ of 1 's in the first $n$ digits of the dyadic expansion for $x$. Define

$$
f(x)= \begin{cases}\lim _{n \rightarrow \infty} \frac{N[1, n, x]}{n} & \text { if it exists } \\ 1 & \text { otherwise }\end{cases}
$$

This function allows us to visualize the multifractal components of $[0,1]$ as level sets. If we let $\mu_{p}$ denote the invariant multifractal measure on $K_{p}$ then it is clear that $\int f(y) \mathrm{d} \mu_{p}(y)=p$.

In the next section, we state and prove our main result, and finally we close with some open problems.

\section{Main Result}

We will need the following result by Besicovitch and Moran. This is not the form in which it was originally stated and proved. However, this modern version may be found in [12].

Theorem 1. For any $s \in(0,1)$ there exists a constant $b_{s}>0$ such that for all Borel sets $E \subseteq \mathbf{R}^{2}$ we must have

$$
\mathcal{H}^{1+s}(E) \geq b_{s} \int \mathcal{H}^{s}\left(E_{y}\right) \mathrm{d} y
$$

where $E_{y}=\{x:(x, y) \in E\}$.

We are now ready to state the main result:

Theorem 2. Let $B=\operatorname{Graph}(f) \subseteq[0,1] \times[0,1]$. Then $\operatorname{dim}_{H} B=2$ and $\mathcal{H}^{2}(B)=0$.

Proof. The upper bound is obvious while the lower bound follows from Theorem 1. Fix $s \in(0,1)$ and choose $p$ such that $\frac{p \log p+q \log q}{-\log 2}>s$. Therefore $\mathcal{H}^{s}\left(f^{-1}(\{p\})\right)=\infty$. We can choose an interval $I_{s}$ containing $p$ such that $\mathcal{H}^{s}\left(B_{y}\right)=\infty$ for every $y \in I_{s}$. It follows from Theorem 1 that $\mathcal{H}^{1+s}(B)=\infty$. Observing that $s<1$ was arbitrary gives us the lower bound. Moreover, since every vertical line meets $B$ exactly once, Fubini's theorem tells us that in fact $\mathcal{H}^{2}(B)=0$.

\section{Concluding Remarks}

Here we pose some problems related to the BesicovitchEggleston function. 
1) Can one find the precise scaling function $\phi$ such that $0<\mathcal{H}^{\phi}(B)<\infty$ ? Is the set $[0,1] \times[0,1] \backslash B$ immesaurable, that is, either null or non- $\sigma$-finite for every translation invariant Borel measure on $\mathbf{R}^{2}$ ? See [10], where it is shown that the set $\mathbf{R} \backslash \bigcup_{0<p<1 / 2} K_{p}$ is immeasurable.

2) What is the relationship between $f(x)$ and $f^{2}(x)$ ?

3) How large is the set of points $x$ such that $x=f(x)$ ? Can we characterize this set of fixed points?

\section{References}

[1] L. Barreira, B. Saussol and J. Schmeling, "Distribution of Frequencies of Digits via Multifractal Analysis,” Journal of Number Theory, Vol. 97, No. 2, 2002, pp. 410-438. doi:10.1016/S0022-314X(02)00003-3

[2] A. Besicovitch, "On the Sum of Digits of Real Numbers Represented in the Dyadic System," Mathematische Annalen, Vol. 110, No. 1, 1934, pp. 321-330. doi:10.1007/BF01448030

[3] P. Billingsley, "Hausdorff Dimension in Probability Theory II,” Illinois Journal of Mathematics, Vol. 5, No. 2, 1961, pp. 291-298.

[4] H. Cajar, "Billingsley Dimension in Probability Spaces," Springer-Verlag, Berlin-New York, 1981.

[5] C. S. Dai and S. J. Taylor, "Defining Fractals in a Probability Space,” Illinois Journal of Mathematics, Vol. 38, No. 3 1994, pp. 480-500.

[6] M. Das, "Billingsley’s Packing Dimension,” Proceedings of the American Mathematical Society, Vol. 136, No. 1, 2008, pp. 273-278. doi:10.1090/S0002-9939-07-09069-7

[7] M. Das, "Hausdorff Measures, Dimensions and Mutual Singularity," Transactions of the American Mathematical
Society, Vol. 357, No. 11, 2005, pp. 4249-4268. doi:10.1090/S0002-9947-05-04031-6

[8] H. G. Eggleston, "The Fractional Dimension of a Set Defined by Decimal Properties," Quarterly Journal of Mathematics-Oxford Journals, Vol. 2, No. 20, 1949, pp. 31-36.

[9] G. A. Edgar, "Measure, Topology, and Fractal Geometry,” Springer-Verlag, New York, 1990.

[10] M. Elekes and T. Keleti, "Borel Sets which are Null or Non- $\sigma$-Finite for Every Translation Invariant Measure,” Advances in Mathematics, Vol. 201, No. 1, 2006, pp. 102-115. doi:10.1016/j.aim.2004.11.009

[11] K. J. Falconer, “Techniques in Fractal Geometry,” John Wiley \& Sons, Ltd., Chichester, 1997.

[12] K. J. Falconer, "The Geometry of Fractal Sets," Cambridge University Press, Cambridge, 1986.

[13] A. H. Fan, L. M. Liao, J. H. Ma and B. W. Wang, "Dimension of Besicovitch-Eggleston Sets in Countable Symbolic Space,” Nonlinearity, Vol. 23, No. 5, 2010, pp. 1185-1197.

[14] L. Olsen, "On the Hausdorff Dimension of Generalized Besicovitch-Eggleston Sets of $d$-Tuples of Numbers," Indagationes Mathematicae, Vol. 15, No. 4, 2004, pp. 535-547. doi:10.1016/S0019-3577(04)80017-X

[15] L. Olsen, “Applications of multifractal divergence points to some sets of $d$-tuples of numbers defined by their $N$-adic expansion," Bulletin des Sciences Mathématiques, Vol. 128, No. 4, 2004, pp. 265-289.

[16] L. Olsen, “Applications of Multifractal Divergence Points to Sets of Numbers Defined by Their $N$-Adic Expansion," Mathematical Proceedings of the Cambridge Philosophical Society, Vol. 136, No. 1, 2004, pp. 139-165. doi:10.1017/S0305004103007047 